\title{
Evaluation of Vector-Borne Disease Intervention Strategies Using Compartmental Models: A Case Study of 2016 Zika Epidemic In Miami-Dade County, Florida
}

Nana Luo ( $\square$ luonana@bucea.edu.cn )

Beijing University of Civil Engineering and Architecture School of Architecture and Urban Planning https://orcid.org/0000-0002-5114-0308

\section{Susan Cassels}

University of California Santa Barbara

\section{Atsushi Nara}

San Diego State University

\section{Zhanfeng Shen}

Chinese Academy of Sciences

\section{Research article}

Keywords: Disease Control and Prevention, SEIR Compartmental Model, Vector-borne Disease Intervention Strategies, Zika

Posted Date: August 10th, 2021

DOI: https://doi.org/10.21203/rs.3.rs-790264/v1

License: (1) (i) This work is licensed under a Creative Commons Attribution 4.0 International License.

Read Full License 
1 Evaluation of Vector-borne Disease Intervention Strategies Using Compartmental

2 Models: A Case Study of 2016 Zika Epidemic in Miami-Dade County, Florida

Nana Luo ${ }^{1 *}$, Susan Cassels ${ }^{2}$, Atsushi Nara ${ }^{3,4}$, Zhanfeng Shen ${ }^{5,6}$

1. School of Geomatics and Urban Spatial Informatics, Beijing University of Civil Engineering and Architecture, Beijing, China

2. Department of Geography, University of California-Santa Barbara, Santa Barbara, 93106, CA, USA

3. Department of Geography, San Diego State University, San Diego, 92182, CA, USA

4. Center for Human Dynamics in the Mobile Age, San Diego State University, San Diego, 92182, CA, USA

5. Aerospace Information Research Institute, Chinese Academy of Sciences, Beijing 100101, China

6. College of Resources and Environment, University of Chinese Academy of Sciences, Beijing 100049, China

*Corresponding author: luonana@bucea.edu.cn

\begin{abstract}
Background: In August 2016, Miami-Dade County implemented Zika intervention strategies to combat a dramatic increase in incidence. Encouragingly, there was a significant decrease; howbeit, the effectiveness of these interventions remains unclear, and many countries in the world still suffer from various vector-borne diseases.

Methods: To evaluate emerging vector-borne disease intervention strategies, we propose a Susceptible-Exposed-Infectious-Recovered Intervention Model (SEIR-IM) and apply the model to the 2016 Zika outbreak in Miami-Dade County, Florida as a case study. The proposed model allows for the impacts of the interventions on the
\end{abstract}


transmission cycle of vector-borne diseases, and is parameterized by the Markov Chain Monte Carlo (MCMC).

Results: Within the exiting literature, we find that vector-borne disease intervention strategies promote disease control in the context of human, vector, and human-vector contact. Overall, during the course of interventions, the transmission probability of humans decreases from 0.417 to 0.38 versus mosquitoes from 0.418 to 0.19 . Through further analysis, the host-based controls are able to reduce the human infections to 27 , while the vector-based controls are 50. On the basis of analysis of the human infections at high intervention coverage, in particular, continued scale-up of the interventions from baseline, we find that the interventions at higher coverage lead to an earlier but higher infection peak: 20 more infections and 100 days in advance.

Conclusions: The three interventions result in a remarkable decline of the Zika infection in Miami-Dade County, and the host-based and contact- based controls are the most effective in reducing Zika incidence.

Keywords: Disease Control and Prevention, SEIR Compartmental Model, Vectorborne Disease Intervention Strategies, Zika

\section{Background}

Vector-borne disease outbreaks are a major threat to global health, economies, and society [1-17]. In the United States, the Zika virus epidemic in 2015-16 resulted in 5,102 cases and the short term economic impact of US\$3.5 billion. Therefore, assessing emerging intervention strategies for vector-borne diseases is extremely important [1828]. We conducted a model-based evaluation of emerging vector-borne disease interventions, using the 2016 Zika epidemic in Miami-Dade County, Florida as a case study. A greater understanding of the intervention strategies that successfully combatted the 2016 Zika epidemic in Miami-Dade County, Florida will aid many countries that remain suffering from vector-borne diseases [4,19,29-34].

In 2016, the United States reported an increase in Zika due to the bites of Aedes mosquitoes, especially in the state of Florida [10]. Miami-Dade County was a hotspot 
for Zika infection, but experienced a dramatic decrease in incidence after countywide truck/aerial adulticide/larvicide outdoor spraying and other CDC-recommended (Centers for Disease Control and Prevention, CDC) controls were implemented. In particular, the CDC suggested environmental interventions such as draining any water containers including garbage cans and toys and discarding old tires and drums. These recommendations reduce the living aquatic habitat of the mosquito larva. They also suggested individual interventions including using mosquito repellent and regular bednets, wearing long clothing, installing screens on windows and doors, and avoiding travelling to mosquito-active areas. These interventions reduce or block contact between humans and adult mosquitoes.

Mathematical models have become well established in the past two decades to assess emerging vector-borne disease intervention strategies including regular nets, artemisinin-combination therapy (ACT), long-lasting insecticide treated nets (LLINs), indoor residual spraying (IRS), mass screening and treatment (MSAT) and vaccination [35-42]. In particular, compartmental models are capable of taking into account these intervention strategies in the transmission cycles of vector-borne diseases and quantify their impacts on disease control and prevention using ordinary differential equations [20,23,36,38, 43-48].

Therefore, the aim of this study is to determine the most effective type of interventions that could combat emerging vector-borne disease outbreaks in the future. To achieve this aim, we propose a Susceptible-Exposed-Infectious-Recovered compartmental intervention model (SERI-IM) that includes the intervention strategies cited above, and apply the model to Miami-Dade County, Florida. We collected data from the 2016 Zika outbreak in Miami-Dade County and evaluated how successful these interventions were by varying their coverage and comparing disease incidence for human hosts. The rest of this paper is organized as follows. Section 2 describes the proposed methodology, specifically an overview of the baseline model, SEIR and intervention model, SEIR-IM as well as model parameterization. Section 3 focuses on the results of applying the proposed model to the 2016 Zika epidemic in Miami-Dade County, Florida, including the human cases predicted from the baseline model, 
intervention model, and intervention evaluation. Section 4 concludes with a summary of findings, discussions and limitations.

\section{METHODS}

\section{Data Source}

In 2016-17, the Florida Department of Health reported daily counts of Zika infection by county across Florida, including travel-related cases, locally-acquired cases, cases among pregnant women and sexual-transmitted cases. Travel-related Zika cases are residents who acquired their infection while outside of Florida, while local cases are residents who were infected locally. The Florida Department of Health used Polymerase Chain Reaction technique (PCR) to identify present virus, and Antibody Capture Enzyme-Linked Immunosorbent Assay technique (IgM Antibody) to identify recent past infection. Next they queried the patients with positive results about their travel history, close contact, sexual history and mosquito contact rate. Finally, the laboratory and interview results determined whether the infection was travel-related, locallyacquired, congenital or sexual-transmitted. In this study we focused on the local transmission of Zika in Miami-Dade County, and collected the locally-acquired cases from 1 August 2016 to 17 May 2017 from the Florida Department of Health.

As the CDC reported, Florida was the only state with locally-transmitted cases during the 2016 Zika outbreak in the United States, and most of those cases were within Miami-Dade County. The first Zika case in Florida was reported on 19th January 2016, and was travel-related. As the count of the travel-related cases increased, the first local case was reported on 1 August 2016 in Miami-Dade County, and triggered a local outbreak across the county.

\section{The Baseline Model, SEIR}

Model Overview. The SEIR model with human hosts, mosquito vectors and virus transmission via interaction and bite, one of the widely used compartmental models, stratifies hosts and vectors into four and three subgroups. The groups are: Susceptible (S), Exposed (E), Infectious (I) and Recovery (R) for the human hosts, and Susceptible 
(S), Exposed (E), and Infectious (I) for the mosquito vectors. To build the baseline model, we speculate that: 1) after getting infected with Zika, humans do not become infectious immediately; 2) people acquire lifelong immunity after recovery; 3) the population is closed; and 4) in a transmission cycle, mosquitoes die before recovery. Although climate factors such as temperature and humidity may influence mosquito populations, spatiotemporal distribution, movement, and reproduction rate [49], the impact would be weakened by the outdoor spraying applied in Miami-Dade County. Such large-scale vector control programs can kill a great deal of vectors on a short time scale. Compared to dramatic and rapid declines in vector population due to outdoor sprayings, the climate- and environment- driven impact becomes inappreciable.

Originally developed by Kucharski et al. [50], Figure 1 depicts the details of the SEIR model. $\beta^{H}$ represents the transmission rate from vectors to humans; $\beta^{V}$ is the transmission rate from humans to vectors; $\alpha^{H}$ and $\alpha^{V}$ are the latent periods for humans and vectors; $\gamma$ stands for the recovery rate of humans; $\delta$ is the lifespan of vectors; $S^{H}, E^{H}, I^{H}$ and $R^{H}$ are the population for susceptible, exposed, infectious and recovery humans; and $S^{V}, E^{V}$ and $I^{V}$ are the population for susceptible, exposed and infectious mosquitoes. Transmission occurs when a susceptible vector bites an infectious host and/or an infectious vector bites a susceptible host, and is formulated as follows:

$$
\begin{gathered}
S^{H}(t+1)=S^{H}(t)-\beta^{H} * S^{H}(t) * I^{V} / N^{H} \\
E^{H}(t+1)=E^{H}(t)+\beta^{H} * S^{H}(t) * I^{V} / N^{H}-E^{H}(t) * \alpha^{H} \\
I^{H}(t+1)=I^{H}(t)+E^{H}(t) * \alpha^{H}-I^{H}(t) * \gamma \\
R^{H}(t+1)=R^{H}(t)+I^{H}(t) * \gamma \\
N^{H}(t)=S^{H}(t)+E^{H}(t)+I^{H}(t)+R^{H}(t) \\
S^{V}(t+1)=S^{V}(t)-\beta^{V} * S^{V}(t) * I^{H} / N^{V}+N^{V}(t) * \delta-S^{V}(t) * \delta \\
E^{V}(t+1)=E^{V}(t)+\beta^{V} * S^{V}(t) * I^{H} / N^{V}-E^{V}(t) * \alpha^{V}-E^{V}(t) * \delta \\
I^{V}(t+1)=I^{V}(t)+E^{V}(t) * \alpha^{V}-I^{H}(t) * \delta \\
N^{V}(t)=S^{V}(t)+E^{V}(t)+I^{V}(t)
\end{gathered}
$$

where $t$ is the current time step; $t+1$ is the next time step; $N^{H}$ is the total population for humans; and $N^{V}$ is the total population for mosquitoes. 


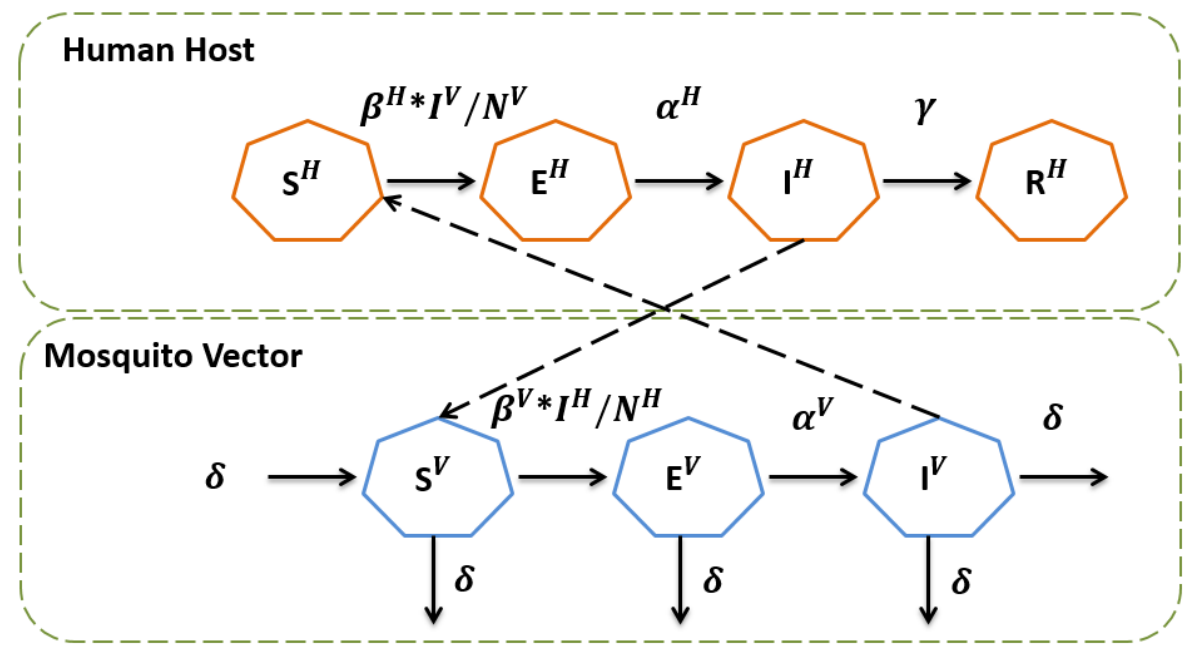

Fig. 1 The baseline model. Human hosts are divided into four compartments, namely susceptible, exposed, infectious and recovered. People who are not currently infected with, but susceptible to Zika are susceptible, people who are infected with Zika but unable to transmit Zika virus are exposed, people who are able to transmit the virus are infectious, and people who recover from Zika disease are recovered. Mosquito vectors are divided into three compartments, including susceptible, exposed and infectious.

Model Parameters. Table 1 lists the values and sources of the parameters in the baseline model. The average latent periods for humans $\alpha^{H}$ and mosquitoes $\alpha^{V}$ are 5.9 days and 10.5 days [51]; the recovery period for humans $\gamma$ is 5 days [52]; the average lifespan of mosquitoes $\delta$ is 15 days [50]; the transmission probabilities from vector to human $\beta^{H}$ and from human to vector $\beta^{V}$ are 0.417 and 0.418 [50].

Table 1. Parameters for the baseline model - SEIR.

\begin{tabular}{c|l|l|l}
\hline Parameter & Definition & Sources & Values \\
\hline$\beta^{H}$ & $\begin{array}{l}\text { Transmission probability of } \\
\text { human host }\end{array}$ & {$[50]$} & 0.417 \\
\hline$\alpha^{H}$ & Latent period of human host & {$[51]$} & 0.169 \\
\hline$\gamma$ & Recovery rate of human host & {$[52]$} & 0.200 \\
\hline$\beta^{V}$ & $\begin{array}{l}\text { Transmission probability of } \\
\text { mosquito vector }\end{array}$ & {$[50]$} & 0.418 \\
\hline$\alpha^{V}$ & Latent period of mosquito vector & {$[51]$} & 0.095 \\
\hline$\delta$ & $\begin{array}{l}\text { Death/Birth rate of mosquito } \\
\text { vector }\end{array}$ & {$[50]$} & 0.128 \\
\hline
\end{tabular}


Model Initials. According to the Florida Department of Health, the local Zika infection in Miami-Dade Country began on 1 August 2016, which is thus the initial date of the baseline modeling exercise. According to the United States Census Bureau, the population in Miami-Dade Country on that day was 2,692,990. Estimating the initial states of the vectors is more difficult. As Kucharski et al. proposed, the exposed and infectious hosts/vectors are initially equal [50]. On 1 August 2016, Miami-Dade County had 99 travel-related infections and 14 local-acquired cases. We speculate that these 99 travel cases were the source of the 14 local infections, and estimate 203 exposed (E) and 203 infectious (I) mosquitos using Eq. (1) to (9).

\section{The Intervention Model, SEIR-IM}

Model Overview. Emerging vector-borne disease intervention strategies reduce disease transmissions by targeting either the human hosts, mosquito vectors, or human-vector contact [35,53-60]. The host-based controls prevent disease transmission by completely separating people from virus; these include regular bed-net usage, wearing long clothing, and avoiding traveling to virus-active areas. The vector-based controls such as outdoor sprayings and LLINs reduce infections by reducing mosquito populations. Lastly, the contact-based controls like mosquito repellent usage prevent transmission by reducing human-vector contact.

According to the nature of these three controls on disease prevention and control, many researchers perform model-based vector-borne disease intervention evaluation by formulating the effect of the interventions in the transmission cycles of the diseases. In Agusto et al., the LLINS control on malaria is a decreasing function of how long people use it since the pesticide treatment on nets loses efficacy due to frequent washing and exposure to sunlight [39]. Griffin et al. speculated that IRS decreases mosquito population or blocks human-mosquito contacts because it kills mosquitoes when they rest on the wall and repel them before they feed [40].

Similarly, we formulated three types of controls including the host-based, vectorbased, and contact-based, as follows: 1) people who use the host-based controls are not susceptible to Zika; 2) the vector-based controls minimize the vector population; and 3) people who use the contact-based controls have a lower human-mosquito contact rate. 
Figure 2 illustrates how we formulate these three interventions in the transmission cycle of Zika virus. The host-based controls reduce the population of the susceptible humans $S^{H}$ by $d^{H}$. The contact-based controls affect the human-vector contact rates $\beta^{H}$ and $\beta^{V}$ to be new, lower values $\beta_{2}^{H}$ and $\beta_{2}^{V}$. The vector-based controls minimize the population of the susceptible vectors $S^{V}$ by $d^{V}$.

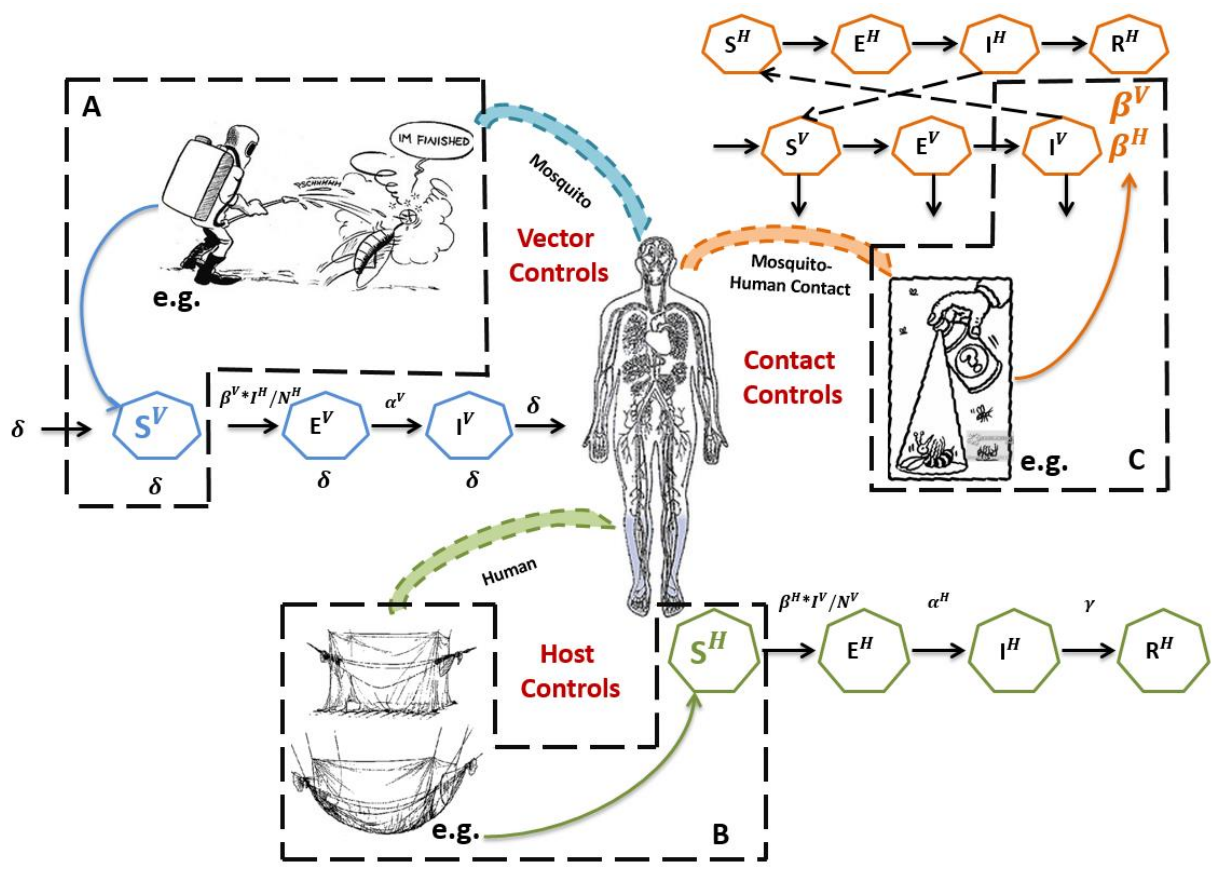

Fig. 2 The intervention model. Building on the baseline model, the intervention model takes into account the vector-based, host-based, and contact-based controls. The vectorbased controls minimize the vector population. The host-based controls separate humans from virus. The contact-based controls reduce the contacts between humans and mosquitoes.

Intervention Parameters. Table 2 lists the values and sources of the parameters required in the SEIR-IM, where the transmission probabilities from vector to human and from human to vector $\beta_{2}^{H}$ and $\beta_{2}^{V}$, and the rates $d^{V}$ and $d^{H}$, by which the populations for the susceptible vectors and susceptible hosts $S^{V}$ and $S^{H}$ decrease due to using the host-based and vector-based controls, are unknown and estimated by fitting the SEIR-IM to our data using the Markov Chain Monte Carlo (MCMC). Therefore, the intervention transmissions Eq. (1), (2), (6) and (7) are formulated as below: 


$$
S^{H}(0)^{\prime}=S^{H}(0)\left(1-d^{H}\right)
$$

$$
E^{H}(t+1)=E^{H}(t)+\beta_{2}^{H} * I^{V} * S^{H}(t)-E^{H}(t) * \alpha^{H}
$$

$$
E^{V}(t+1)=E^{V}(t)+\beta_{2}^{V} * I^{H} * S^{V}(t)-E^{V}(t) * \alpha^{V}-E^{V}(t) * \delta
$$

\begin{tabular}{|c|c|c|c|c|}
\hline Parameters & Definition & Sources & $\begin{array}{l}\text { Values } \\
\text { (After) }\end{array}$ & $\begin{array}{c}\text { Values } \\
\text { (Before) }\end{array}$ \\
\hline$\beta_{2}^{H}$ & $\begin{array}{l}\text { New transmission probability for } \\
\text { humans due to using the contact- } \\
\text { based controls }\end{array}$ & $\begin{array}{c}\text { Model } \\
\text { Fit }\end{array}$ & Estimated & 0.417 \\
\hline$\alpha^{H}$ & Latent period for human & [51] & 0.169 & 0.169 \\
\hline$\gamma$ & Recovery rate for human & {$[52]$} & 0.200 & 0.200 \\
\hline$\beta_{2}^{V}$ & $\begin{array}{l}\text { New transmission probability for } \\
\text { mosquitoes due to using the } \\
\text { contact-based controls }\end{array}$ & $\begin{array}{c}\text { Model } \\
\text { Fit }\end{array}$ & Estimated & 0.418 \\
\hline$\alpha^{V}$ & Latent period for vector & {$[51]$} & 0.095 & 0.095 \\
\hline$\delta$ & Death/Birth rate for vector & {$[50]$} & 0.128 & 0.128 \\
\hline$d^{H}$ & $\begin{array}{l}\text { Percentage of human hosts that } \\
\text { are insusceptible to Zika due to } \\
\text { using the host-based controls }\end{array}$ & $\begin{array}{c}\text { Model } \\
\text { Fit }\end{array}$ & Estimated & NA \\
\hline$d^{V}$ & $\begin{array}{l}\text { Percentage of mosquitoes being } \\
\text { killed by using the vector-based } \\
\text { controls }\end{array}$ & $\begin{array}{c}\text { Model } \\
\text { Fit }\end{array}$ & Estimated & NA \\
\hline
\end{tabular}

Table 2. Parameters for the intervention model - SEIR-IM.

Intervention Evaluation. To evaluate the three types of interventions, we vary the parameters $\beta^{H}, d^{H}$ and $d^{V}$ from baseline. In particular, $\beta^{H}$ ranges from 0.382 to 0.388 by $0.02, d^{H}$ fluctuates from 0.982 to 0.988 by 0.02 , and $d^{V}$ differs from 0.992 to 0.998 by 0.02 . We run the SEIR-IM across a 285 -day period that starts from August $1^{\text {st }} 2016$ for each varied intervention parameter, and compare the simulated disease incidence at day 285 with the Zika data.

\section{MCMC-based Model Parameterization and Fit for the SEIR-IM}

Method Overview. To fit the intervention model and estimate the unknown parameters, including the new transmission probabilities from vector to human and from human to vector $\beta_{2}^{H}$ and $\beta_{2}^{V}$ due to using the contact-based controls, the rate for humans $d^{H}$ 
due to using the host-based controls, and the rate for vectors $d^{V}$ due to using the vector-based controls, we used MCMC. This stochastic approach repeatedly generates random sampling from the potential space of the parameters and summarizes their distributions [61]. This parameterization was performed in two stages. First, we generated Markov chains by searching though the parameter space and identified the most likely search region. Second, we undertook the expectation approximation, summarized the samples and calculated the statistical summary of the sampling [61]. Here we used the maximum likelihood estimation, and the likelihood of the four target parameters is formulated as follows:

$$
P(D \mid \theta)=e^{-\sum_{i=1}^{n}\left(y_{i}-\tilde{y}_{i}\right)^{2}}
$$

where $y_{i}$ is the $i^{\text {th }}$ Zika data, and $\tilde{y}_{i}$ is the $i^{\text {th }}$ modeled data, which are computed from the Eq. (1) - (13). And the posterior distribution of the four target parameters is given:

$$
P(\theta \mid D)=e^{-\sum_{i=1}^{n}\left(y_{i}-\tilde{y}_{i}\right)^{2}} * P(\theta)
$$

The aim of MCMC is to identify the combination of the parameters that minimizes $\sum_{i=1}^{n}\left(y_{i}-\tilde{y}_{i}\right)^{2}$ and fits the data best. Accordingly, $P(D \mid \theta)$ and $P(\theta \mid D)$ needs to be maximized. MCMC starts with an initial user-defined value for the target parameters, computes $P(D \mid \theta)$, searches the next potential values through the parameter space, and calculates new $P(D \mid \theta)$. By comparing these two $P(D \mid \theta)$, we will keep the larger one. We constructed a Markov chain and calculated the summary statistic of the chain.

\section{RESULTS}

\section{Human Infections in the Baseline Simulation}

Results for the Zika infections by day for human hosts in the baseline simulation are shown in Figure 3, where the x-axis stands for the simulated Zika cases, and the y-axis represents the infection cases reported by the Florida Department of Health. Within the simulation, the model and reported data matched well for the first 60 days (1st August 2016 - 30th September 2016); the intervention modeling exercise started on day 61 (1st October 2016. It is noteworthy that Miami-Dade County applied the countywide 
truck/aerial adulticide/larvicide outdoor spraying began around October $1^{\text {st }} 2016$.

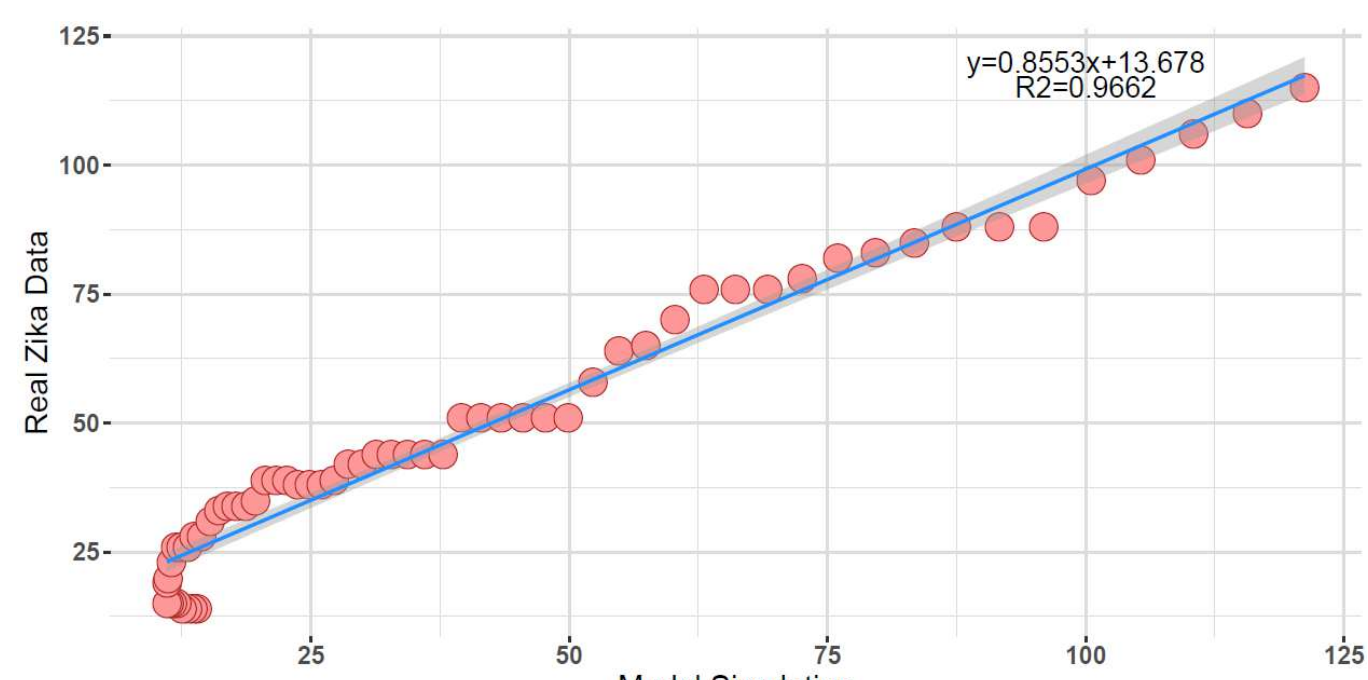

Model Simulation

Fig. 3 The baseline simulation results, in particular, the comparison between the predicted and observed. The $\mathrm{x}$-axis is the simulated data, and the $\mathrm{y}$-axis is the reported data.

Figure 4 depicts the timeline of the 2016 Miami-Dade County Zika epidemic modeling exercise in this study. Although we collect data from Jan 192016 to May 12 2017, the local outbreak began in 1 August 2016 when our baseline simulation starts, and October 2016 is the start date of our intervention modeling exercise.

Jan $19^{\text {th }} 2016$

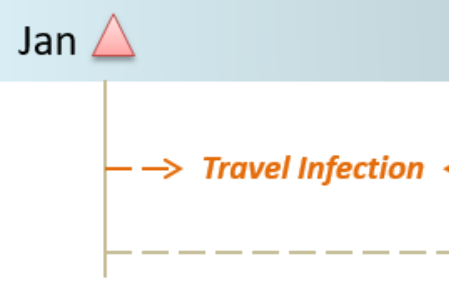

Aug $1^{\text {st }} 2016$ Sep $30^{\text {th }} 2016$

May $12^{\text {th }} 2017$

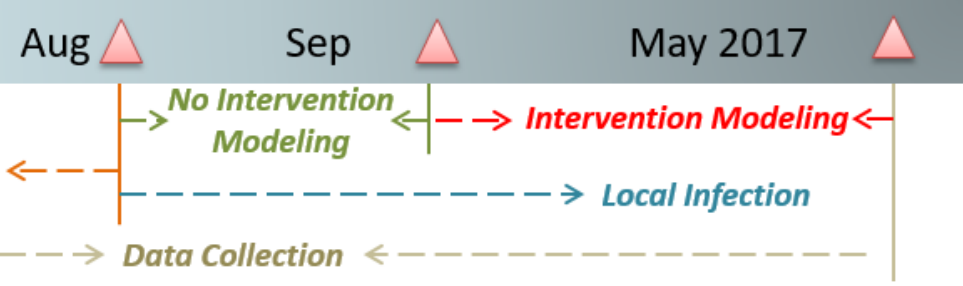

Fig. 4 The timeline of the 2016 Zika epidemic in Miami-Dade County, Florida.

\section{Human Infections in the Intervention Simulation}

Results for the model fit and parameter estimation of the SEIR-IM are shown in Figure 5, which are the well-constructed Markov chains for $\beta^{H}, \beta^{V}, d^{H}$ and $d^{V}$. These four chains converge to the parameter space quickly and display a stable sampling, and the four parameters $\beta^{H}, \beta^{V}, d^{H}$ and $d^{V}$ are estimated to be $0.39,0.18,0.98$ and 0.99 . 

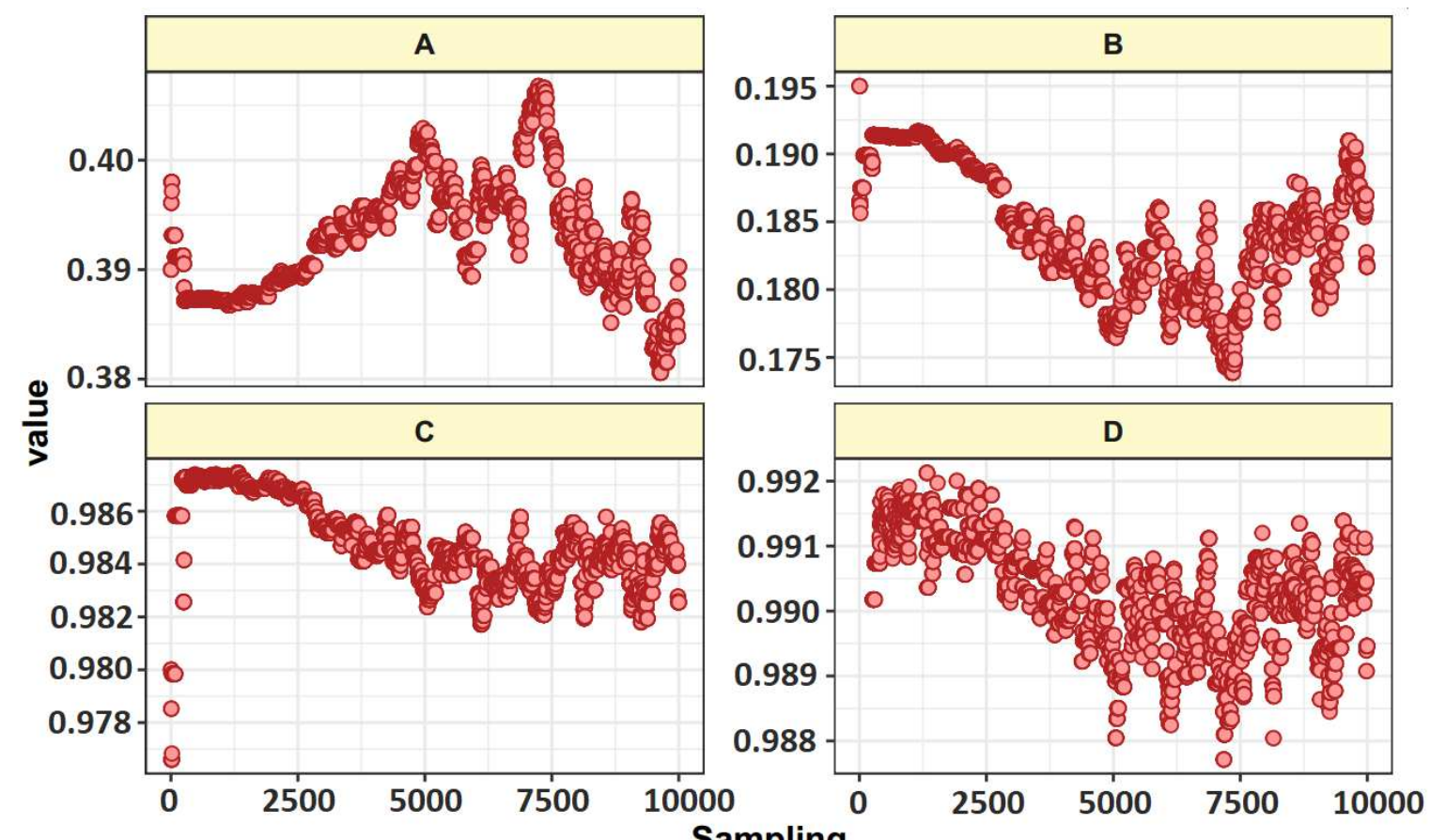

Fig. 5 The MCMC samplings for the intervention modeling parameters $\left(\mathrm{A}: \beta^{H}\right.$ the new transmission probability for humans due to using the contact-based controls; B: $\beta^{V}$ the new transmission probability for mosquitoes due to using the contact-based controls; $\mathrm{C}: d^{H}$ the percentage of humans who are insusceptible to Zika due to using the host-based controls; and D: $d^{V}$ the percentage of mosquitoes being killed by using the vector-based controls).

Results for the Zika infections by day for human hosts in the intervention simulation are shown in Figure 6. While the data reaches the peak around at day 150 versus the peak around at day 180 in our simulation, the model clearly tallies with the data. We also find that the three interventions result in a remarkable decline of the Zika infection in Miami-Dade County, as reported by the daily-updated cases from the Florida Department of Health. 


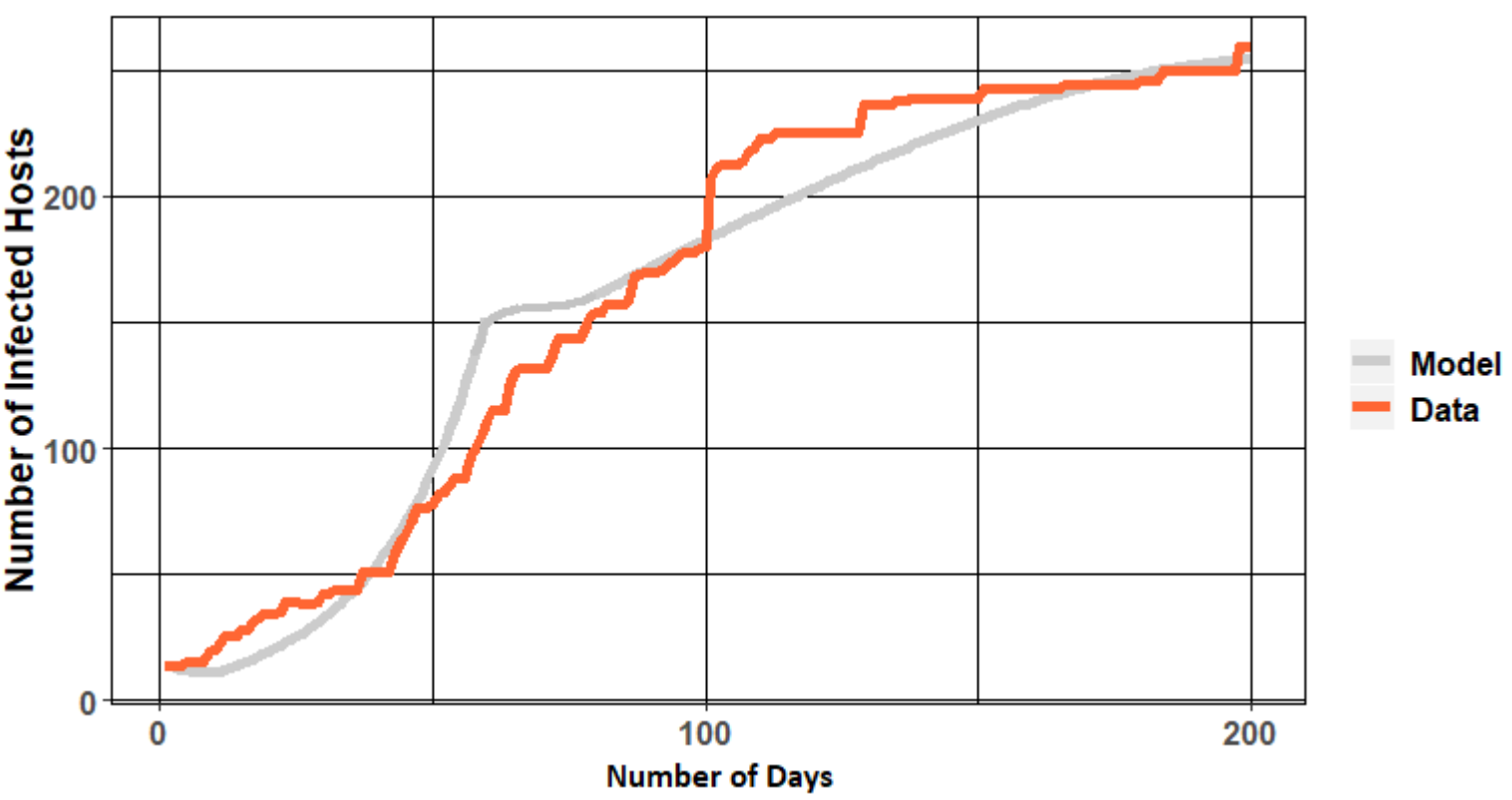

Fig. 6 The intervention modeling results, where the $\mathrm{x}$ axis is the number of days starting from 1 August 2016, and the y axis is the accumulated cases of Zika infection. The grey line is the simulated data, and the orange line is the reported data. Evaluation of the Zika Intervention Strategies

Figure 7 depicts the simulated Zika cases at day 285 for the three types of interventions. We find that increasing intervention coverage is effective in reducing Zika infections, that is, coverage is important to intervention success. Specifically, the number of Zika cases drop dramatically as $\beta^{H}$ or $d^{V}$ decreases, or $d^{H}$ increases. With an increasing $d^{H}$, the number of Zika cases at day 285 declines due to fewer susceptible hosts. In contract, Zika cases increase with an increasing $\beta^{H}$ or $d^{V}$. Through further analysis, the host-based and contact-based controls are able to reduce human infections to 27 , while the vector-based controls are 50 . We can clearly see that the host-based and contact-based controls are effective to minimize human infections. We also find that increasing intervention coverage may reduce disease incidence peak but extend epidemic interval, i.e., large intervention coverage may just delay infections. In Figure 8 , our contact-based simulation peak $\left(\beta^{H}=0.388\right)$ has 20 more infections but approximately 100 days in advance compared to the curve with the $0.382 \beta^{H}$. It confirms findings by others-e.g., the ongoing, novel severe acute respiratory syndrome coronavirus 2 (SARS-CoV-2) outbreak [62]. A delay in peak infections may still be beneficial, as extra time allows hospitals and health system leaders to respond 
to disease such as proving homeless and meal service, allocating resources on 309 organizational preparedness and capacity planning, and considering alternative health care.

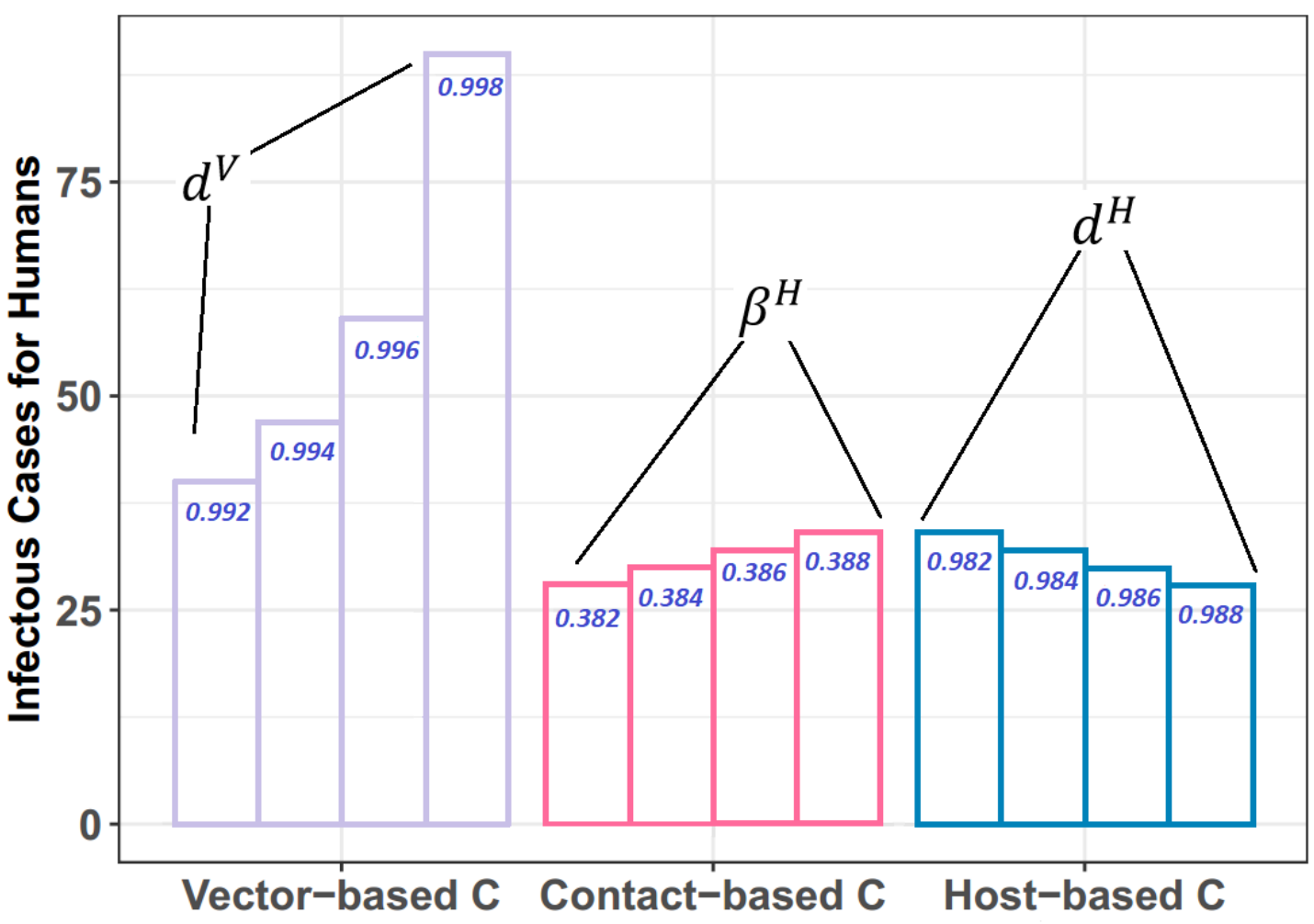

312 Fig. 7 The intervention evaluation results, where the $\mathrm{x}$ axis represents an evaluated 313 intervention including the vector-based controls $\left(d^{V}\right)$, the contact-based controls $314\left(\beta^{H}\right)$, and the host-based controls $\left(d^{H}\right)$, the bar is a specific value for the interventions 315 (i.e., the values for the intervention parameters), and the y is the Zika incidence for day 316285 simulated by the SEIR-IM. 


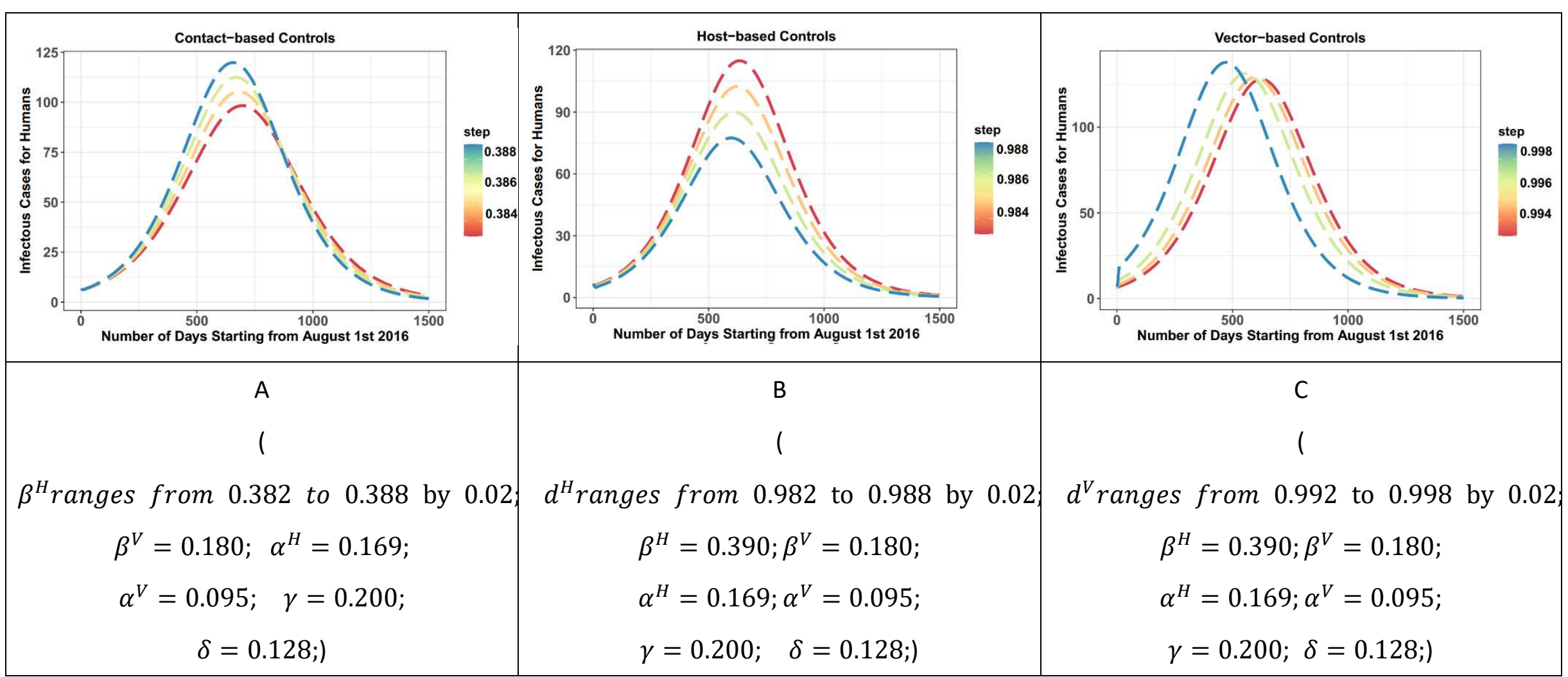

Fig. 8 The intervention evaluation results across a 1500-day period that starts from August $1^{\text {st }} 2016$ (A: the new transmission probability from

vectors to humans $\quad \beta^{H}$ due to using the contact-based controls; B: $d^{H}$ the percentage of human hosts who are insusceptible to Zika due to using the host-based controls; and C: $d^{V}$ the percentage of mosquito vectors being killed by using the vector-based controls). 


\section{Discussions}

321 Within the existing literature, we find that emerging vector-borne disease intervention strategies include three types. They are the host-based controls, vector-based controls, and contact-based controls. The host-based controls aim to separate people from virus; the vector-based controls aim to minimize vector; and the contact-based controls aim to reduce human-vector contact. For vector-borne diseases the host-based and contactbased controls are the most effective in minimizing human infections. We also recommend other similar controls such as wearing long clothing, using screens on windows and doors, and avoiding travelling to mosquito-active areas.

Using MCMC, we estimated the intervention parameters $\beta_{2}^{H}, \beta_{2}^{V}, d^{H}$, and $d^{V}$ to be $0.39,0.18,0.98$ and 0.99 . Although 0.98 usage of the host controls in Miami-Dade County seems overestimated, it is modeled as a representative of all interventions sharing the nature of the bed-net usage for vector-borne diseases control and prevention, for instance, wearing long-sleeved shirts and long pants, using screens on windows and doors, and avoiding traveling to virus-active areas. In general, the proportion of the humans who are insusceptible to Zika due to using the host-based controls, regardless of with or without awareness to completely avoid contact with virus, are $98 \%$.

Our results also confirm findings by others that compartmental models can depict the macro-scale transmission dynamics of diseases based on data accessible at an aggregate scale and revealing valuable general characteristics of the diseases $[63,64$, 40]. Within the simulation, we recognize one general characteristics of vector-borne diseases modeling. At the early stage of an outbreak, few people utilize interventions. In this case, the simplified assumptions of the compartmental models are reasonable to simulate an epidemic. As disease spreads locally and widely, government begins applying public interventions, and people start using individual interventions. At that time, the baseline model fails to understand the disease, and parameters regarding the interventions must be included in order to achieve the simulation. Another reason is that vector-borne diseases have distinctive lifestyles and transmission cycles in comparison 
to other diseases, which makes the disease spread complicated and unpredictable. Additional factors such as the biology of the vector itself, host immunity, geography and climate must be considered. This is also why the SEIR-IM cannot perform longterm prediction.

Whilst detailed comparison of emerging vector-borne disease interventions, for instance, reduction in adherence of the interventions, distributions of the interventions, and population structure, is beyond the scope of this paper, our results successfully demonstrate that coverage is an important determinant of intervention success (65-68). Indeed, high coverage, in particular, continued scale-up of the interventions from baseline, could potentially reduce transmissions given that the dominant vector species considered in this study are both endophagic and exophagic and their biting occurs at any time of a day, even at night. However, we pay little attention to heterogeneous mixing in the targeted population as well as individual heterogeneity and stochasticity in infection probability. Our model is incapable of capturing heterogeneity and stochasticity among pathogens, hosts and vectors in respect to climate, biological, geographical, behavioral, social, economic and environmental variables at a local scale.

\section{Conclusions}

In this study we proposed a novel SEIR intervention model to evaluate emerging interventions to combat vector-borne diseases, and applied the model to the 2016 Zika epidemic in Miami-Dade County, Florida as a case study. Despite these limitations mentioned above, our model provides an appropriate tool for a range of stakeholders to explore the potentials of emerging vector-borne disease interventions and helps to determine the optimal policy for elimination of the diseases. Due to the convenient simplified assumptions, our model also requires less in computation ability and memory, and has good performance in parameter estimation, and model parameterization and fit. These ultimately lead to the built SEIR-IM with potential goal of global eradication of vector-borne diseases. 


\begin{abstract}
Abbreviations
Susceptible-Exposed-Infectious-Recovered Intervention Model: SEIR-IM; Markov Chain Monte Carlo: MCMC; Centers for Disease Control and Prevention: CDC; artemisinin-combination therapy: ACT; long-lasting insecticide treated nets: LLINs; indoor residual spraying: IRS; mass screening and treatment: MSAT; Polymerase Chain Reaction technique: PCR; Antibody Capture EnzymeLinked Immunosorbent Assay technique: IgM Antibody;
\end{abstract}

\title{
Acknowledgement
}

The authors thank the Florida Department of Health for making the Zika dataset freely available that enabled us to prepare this manuscript.

\section{Author contributions}

Dr. Nana Luo and Dr. Susan Cassels designed the study, and wrote the main manuscript text. Dr. Atsushi Nara, and Dr. Zhanfeng Shen provided the technical and editing assistance. All authors reviewed the manuscript.

\section{Funding}

This work was funded from the National Key Research and Development Project of China (2017YFB0504204) and the National Natural Science Foundation of China (41971375) at Chinese Academy of Sciences, and the Hellman Family Faculty Fellows Program at the University of California Santa Barbara.

\section{Data Availability}

The datasets collected and analyzed for the study are publicly available.

\section{Declarations}

\section{Ethics approval and consent to participate}

The data used for this manuscript are available in the public domain. Therefore, an ethical approval was not required for this manuscript preparation.

\section{Competing interests}

The authors declare to have no conflicts of interest and no competing interests.

\section{Consent to participate}

Not applicable.

\section{Reference}

1. Chan EYY, Lo ESK, Huang Z, Lam HCY, Yeung MP, Kwok K, Hung KK, Tse SL. Sociodemographic predictors of knowledge, mosquito bite patterns and protective behaviors concerning vector borne disease: The case of dengue fever in Chinese subtropical city, Hong Kong. PLoS Negl Trop Dis. 2021; 15: e0008993. https://doi.org/10.1371/journal.pntd.0008993

2. Nosrat C, Altamirano J, Anyamba A, Caldwell JM, Damoah R, Mutuku F, Ndenga B, LaBeaud AD. Impact of recent climate extremes on mosquito-borne disease transmission in Kenya. PLoS Negl Trop Dis. 2021; 15: e0009182. doi: 10.1371/journal.pntd.0009182.

3. Karuppusamy B, Sarma DK, Lalmalsawma P, Pautu L, Karmodiya K, Balabaskaran Nina P. Effect of climate change and deforestation on vector borne diseases in the North-Eastern Indian State of Mizoram bordering Myanmar. J Clim Change Health. 2021; 2: 100015. 
https://doi.org/10.1016/j.joclim.2021.100015

4. Cao-Lormeau V-M, Blake A, Mons S, Lastère S, Roche C, Vanhomwegen J, Dub T, Baudouin L, Teissier A, Larre P, Vial AL. Guillain-Barré Syndrome outbreak associated with Zika virus infection in French Polynesia: a case-control study. The Lancet. 2016; 387: 1531-9. https://doi.org/10.1016/S0140-6736(16)00562-6

5. Cauchemez S, Besnard M, Bompard P, Dub T, Guillemette-Artur P, Eyrolle-Guignot D, Salje H, Van Kerkhove MD, Abadie V, Garel C, Fontanet A. Association between Zika virus and microcephaly in French Polynesia, 2013-15: a retrospective study. The Lancet. 2016;387: 2125-32.

6. Eisen RJ, Kugeler KJ, Eisen L, Beard CB, Paddock CD. Tick-Borne Zoonoses in the United States: Persistent and Emerging Threats to Human Health. ILAR J. 2017; 58: 319-35.

7. Flores HA, O’Neill SL. Controlling vector-borne diseases by releasing modified mosquitoes. Nat Rev Microbiol. 2018; 16: 508-18.

8. Gubler D. Resurgent Vector-Borne Diseases as a Global Health Problem. Emerg Infect Dis. 1998; 4: 442-50.

9. Heukelbach J, Werneck GL. Surveillance of Zika virus infection and microcephaly in Brazil. The Lancet. 2016; 388: 846-7.

10. Malone RW, Homan J, Callahan MV, Glasspool-Malone J, Damodaran L, Schneider ADB, Zimler R, Talton J, Cobb RR, Ruzic I, Smith-Gagen J. Zika Virus: Medical Countermeasure Development Challenges. Kading RC, editor. PLoS Negl Trop Dis. 2016; 10: e0004530.

11. Mlakar J, Korva M, Tul N, Popović M, Poljšak-Prijatelj M, Mraz J, Kolenc M, Resman Rus K, Vesnaver Vipotnik T, Fabjan Vodušek V, Vizjak A. Zika Virus Associated with Microcephaly. N Engl J Med. 2016; 374: 951-8.

12. Mysorekar IU, Diamond MS. Modeling Zika Virus Infection in Pregnancy. Phimister EG, editor. N Engl J Med. 2016; 375: 481-4.

13. Petersen LR, Beard CB, Visser SN. Combatting the Increasing Threat of Vector-Borne Disease in the United States with a National Vector-Borne Disease Prevention and Control System. Am J Trop Med Hyg. 2019; 100: 242-5.

14. Rasmussen SA, Jamieson DJ, Honein MA, Petersen LR. Zika Virus and Birth Defects Reviewing the Evidence for Causality. N Engl J Med. 2016; 374: 1981-7.

15. Shaw WR, Catteruccia F. Vector biology meets disease control: using basic research to fight vector-borne diseases. Nat Microbiol. 2019; 4: 20-34.

16. Swei A, Couper LI, Coffey LL, Kapan D, Bennett S. Patterns, Drivers, and Challenges of Vector-Borne Disease Emergence. Vector-Borne Zoonotic Dis. 2020; 20: 159-70.

17. van den Berg H, Manuweera G, Konradsen F. Global trends in the production and use of DDT for control of malaria and other vector-borne diseases. Malar J. 2017; 16: 401.

18. Wilson AL, Courtenay O, Kelly-Hope LA, Scott TW, Takken W, Torr SJ, Lindsay SW. The importance of vector control for the control and elimination of vector-borne diseases. Barrera 
R, editor. PLoS Negl Trop Dis. 2020; 14: e0007831.

19. N’Dri BP, Heitz-Tokpa K, Chouaïbou M, Raso G, Koffi AJ, Coulibaly JT, Yapi RB, Müller P, Utzinger J. Use of Insecticides in Agriculture and the Prevention of Vector-Borne Diseases: Population Knowledge, Attitudes, Practices and Beliefs in Elibou, South Côte d'Ivoire. Trop Med Infect Dis. 2020; 5: 36.

20. Majumder S, Rahman T, Sigamani A, Hossan S. Therapeutic and Alternate Preventive Measures to Combat Dengue Epidemic in the Indian Subcontinent. 2020; 16.

21. Bañuelos S, Martinez MV, Mitchell C, Prieto-Langarica A. Using mathematical modelling to investigate the effect of the sexual behaviour of asymptomatic individuals and vector control measures on Zika. Lett Biomath. 2019; 6: 1-19.

22. Marcos-Marcos J, Olry de Labry-Lima A, Toro-Cardenas S, Lacasaña M, Degroote S, Ridde V, Bermudez-Tamayo C. Impact, economic evaluation, and sustainability of integrated vector management in urban settings to prevent vector-borne diseases: a scoping review. Infect Dis Poverty. 2018; 7: 83.

23. Padmanabhan P, Seshaiyer P, Castillo-Chavez C. Mathematical modeling, analysis and simulation of the spread of Zika with influence of sexual transmission and preventive measures. Lett Biomath. 2017; 4: 148-66.

24. Rodriguez-Morales AJ, García-Loaiza CJ, Galindo-Marquez ML, Sabogal-Roman JA, MarinLoaiza S, Lozada-Riascos CO, Diaz-Quijano FA. Zika infection GIS-based mapping suggest high transmission activity in the border area of La Guajira, Colombia, a northeastern coast Caribbean department, 2015-2016: Implications for public health, migration and travel. Travel Med Infect Dis. 2016; 14: 286-8.

25. Gao D, Lou Y, He D, Porco TC, Kuang Y, Chowell G, Ruan S. Prevention and Control of Zika as a Mosquito-Borne and Sexually Transmitted Disease: A Mathematical Modeling Analysis. Sci Rep. 2016; 6: 28070.

26. Musso D, Cao-Lormeau VM, Gubler DJ. Zika virus: following the path of dengue and chikungunya? The Lancet. 2015 ; 386: 243-4.

27. Beltrame A, Zammarchi L, Zuglian G, Gobbi F, Angheben A, Marchese V, Degani M, Mantella A, Bianchi L, Montagnani C, Galli L. Schistosomiasis Screening of Travelers from Italy with Possible Exposure in Corsica, France. Emerg Infect Dis. 2015; 21: 1887-9.

28. Bogoch II, Brady OJ, Kraemer MUG, German M, Creatore MI, Kulkarni MA, Brownstein JS, Mekaru SR, Hay SI, Groot E, Watts A. Anticipating the international spread of Zika virus from Brazil. The Lancet. 2016; 387: 335-6.

29. Cruvinel VRN, Zolnikov TR, Takashi Obara M, Oliveira VTL de, Vianna EN, Santos FSG do, de Oliveira KC, Scott JA. Vector-borne diseases in waste pickers in Brasilia, Brazil. Waste Manag. 2020; 105: 223-32.

30. Zhao S, Musa SS, Hebert JT, Cao P, Ran J, Meng J, He D, Qin J. Modelling the effective reproduction number of vector-borne diseases: the yellow fever outbreak in Luanda, Angola 
2015-2016 as an example. PeerJ. 2020; 8: e8601.

31. Caminade C, McIntyre KM, Jones AE. Impact of recent and future climate change on vectorborne diseases: Climate change and vector-borne diseases. Ann N Y Acad Sci. 2019; 1436: $157-73$.

32. Grillet ME, Hernández-Villena JV, Llewellyn MS, Paniz-Mondolfi AE, Tami A, VincentiGonzalez MF, Marquez M, Mogollon-Mendoza AC, Hernandez-Pereira CE, Plaza-Morr JD, Blohm G. Venezuela's humanitarian crisis, resurgence of vector-borne diseases, and implications for spillover in the region. Lancet Infect Dis. 2019; 19: e149-61.

33. Campos GS, Bandeira AC, Sardi SI. Zika Virus Outbreak, Bahia, Brazil. Emerg Infect Dis. $2015 ; 21: 1885-6$.

34. Hayes EB. Zika Virus Outside Africa. Emerg Infect Dis. 2009; 15: 1347-50.

35. Bi K, Chen Y, (John) Wu C-H, Ben-Arieh D. A memetic algorithm for solving optimal control problems of Zika virus epidemic with equilibriums and backward bifurcation analysis. Commun Nonlinear Sci Numer Simul. 2020; 84: 105176.

36. Biswas SK, Ghosh U, Sarkar S. Mathematical model of zika virus dynamics with vector control and sensitivity analysis. Infect Dis Model. 2020; 5: 23-41.

37. Counotte MJ, Althaus CL, Low N, Riou J. Impact of age-specific immunity on the timing and burden of the next Zika virus outbreak [Internet]. Epidemiology. 2019. http://biorxiv.org/lookup/doi/10.1101/661223

38. Miyaoka TY, Lenhart S, Meyer JFCA. Optimal control of vaccination in a vector-borne reaction-diffusion model applied to Zika virus. J Math Biol. 2019; 79: 1077-104.

39. Agusto FB, Del Valle SY, Blayneh KW, Ngonghala CN, Goncalves MJ, Li N, Zhao R, Gong H. The impact of bed-net use on malaria prevalence. J Theor Biol. 2013; 320: 58-65.

40. Griffin JT, Hollingsworth TD, Okell LC, Churcher TS, White M, Hinsley W, Bousema T, Drakeley CJ, Ferguson NM, Basáñez MG, Ghani AC. Reducing Plasmodium falciparum Malaria Transmission in Africa: A Model-Based Evaluation of Intervention Strategies. Krishna S, editor. PLoS Med. 2010; 7: e1000324.

41. Burattini MN, Chen M, Chow A, Coutinho FAB, Goh KT, Lopez LF, Ma S, Massad E. Modelling the control strategies against dengue in Singapore. Epidemiol Infect. 2008; 136: 309-19.

42. Bhattarai A, Ali AS, Kachur SP, Mårtensson A, Abbas AK, Khatib R, Al-Mafazy AW, Ramsan M, Rotllant G, Gerstenmaier JF, Molteni F. Impact of Artemisinin-Based Combination Therapy and Insecticide-Treated Nets on Malaria Burden in Zanzibar. White N, editor. PLoS Med. 2007; 4: e309.

43. Goswami NK, Srivastav AK, Ghosh M, Shanmukha B. Mathematical modeling of zika virus disease with nonlinear incidence and optimal control. J Phys Conf Ser. 2018; 1000: 012114.

44. Ajelli M, Moise IK, Hutchings TCSG, Brown SC, Kumar N, Johnson NF, Beier JC. Host outdoor exposure variability affects the transmission and spread of Zika virus: Insights for 
epidemic control. Althouse B, editor. PLoS Negl Trop Dis. 2017; 1: e0005851.

45. Bonyah E, Khan MA, Okosun KO, Islam S. A theoretical model for Zika virus transmission. Kaderali L, editor. PLOS ONE. 2017; 12: e0185540.

46. Funk S, Kucharski AJ, Camacho A, Eggo RM, Yakob L, Murray LM, Edmunds WJ. Comparative Analysis of Dengue and Zika Outbreaks Reveals Differences by Setting and Virus. PLoS Negl Trop Dis. 2016; 10: e0005173.

47. Yang HM. Malaria transmission model for different levels of acquired immunity and temperature-dependent parameters (vector). Rev Saúde Pública. 2000; 34: 223-31.

48. Aron JL. Mathematical modeling of immunity to malaria. Math Comput Model. 1989; 12: 1180.

49. Monaghan AJ, Morin CW, Steinhoff DF, Wilhelmi O, Hayden M, Quattrochi DA, Reiskind M, Lloyd AL, Smith K, Schmidt CA, Scalf PE, Ernst K. On the Seasonal Occurrence and Abundance of the Zika Virus Vector Mosquito Aedes Aegypti in the Contiguous United States. PLoS Curr. 2016. http://currents.plos.org/outbreaks/article/on-the-seasonal-occurrence-andabundance-of-the-zika-virus-vector-mosquito-aedes-aegypti-in-the-contiguous-united-states/

50. Kucharski AJ, Funk S, Eggo RM, Mallet H-P, Edmunds WJ, Nilles EJ. Transmission Dynamics of Zika Virus in Island Populations: A Modelling Analysis of the 2013-14 French Polynesia Outbreak. Barker CM, editor. PLoS Negl Trop Dis. 2016; 10:e0004726.

51. Lessler J, Ott CT, Carcelen AC, Konikoff JM, Williamson J, Bi Q, Kucirka LM, Cummings DA, Reich NG, Chaisson LH. Times to key events in Zika virus infection and implications for blood donation: a systematic review. Bull World Health Organ. 2016; 94: 841-9.

52. Naba KG, B. S. Stability and optimal control analysis of Zika virus with saturated incidence rate. Malaya J Mat. 2020; 8: 331-42.

53. Martin E, Medeiros MC, Carbajal E, Valdez E, Juarez JG, Garcia-Luna S, Salazar A, Qualls WA, Hinojosa S, Borucki MK, Manley HA. Surveillance of Aedes aegypti indoors and outdoors using Autocidal Gravid Ovitraps in South Texas during local transmission of Zika virus, 2016 to 2018. Acta tropica. 2019; 192: 129-37.

54. Bonyah E, Khan MA, Okosun KO, Gómez-Aguilar JF. On the co-infection of dengue fever and Zika virus. Optim Control Appl Methods. 2019; 40: 394-421.

55. Ngonghala CN, Ryan SJ, Tesla B, Demakovskys LR, Mordecai EA, Murdock CC, Bonds MH. Effects of Temperature on Zika Dynamics and Control. Ecology. 2019. http://biorxiv.org/lookup/doi/10.1101/855072

56. Bouzid M, Brainard J, Hooper L, Hunter PR. Public Health Interventions for Aedes Control in the Time of Zikavirus-A Meta-Review on Effectiveness of Vector Control Strategies. Barrera R, editor. PLoS Negl Trop Dis. 2016; 10: e0005176.

57. Rather IA, Kumar S, Bajpai VK, Lim J, Park Y-H. Prevention and Control Strategies to Counter ZIKA Epidemic. Front Microbiol. 2017; 8. http://journal.frontiersin.org/article/10.3389/fmicb.2017.00305/full

58. Singh RK, Dhama K, Khandia R, Munjal A, Karthik K, Tiwari R, Chakraborty S, Malik YS, 
Bueno-Marí R. Prevention and Control Strategies to Counter Zika Virus, a Special Focus on Intervention Approaches against Vector Mosquitoes-Current Updates. Front Microbiol. 2018; 9: 87 .

59. Hunter P. Challenges and options for disease vector control: The outbreak of Zika virus in South America and increasing insecticide resistance among mosquitoes have rekindled efforts for controlling disease vectors. EMBO Rep. 2016; 17: 1370-3.

60. Phaijoo GR, Gurung DB. Mathematical model on analysis of awareness in controlling dengue disease. International Journal of Advanced Research. 2016; 4: 999-1006.

61. Greenland S. Bayesian perspectives for epidemiological research: I. Foundations and basic methods. Int J Epidemiol. 2006; 35: 765-75.

62. Prem K, Liu Y, Russell TW, Kucharski AJ, Eggo RM, Davies N, Flasche S, Clifford S, Pearson CA, Munday JD, Abbott S. The effect of control strategies to reduce social mixing on outcomes of the COVID-19 epidemic in Wuhan, China: a modelling study. Lancet Public Health. 2020; 5: e261-70.

63. Goswami N, Shanmukha B. A mathematical analysis of Zika virus transmission with optimal control strategies. Computational Methods for Differential Equations. 2021; 9: 117-45.

64. Hay SI, Sinka ME, Okara RM, Kabaria CW, Mbithi PM, Tago CC, Benz D, Gething PW, Howes RE, Patil AP, Temperley WH. Developing global maps of the dominant Anopheles vectors of human malaria. PLoS Med. 2010; 7: e1000209.

65. Smith DL, Hay SI, Noor AM, Snow RW. Predicting changing malaria risk after expanded insecticide-treated net coverage in Africa. Trends Parasitol. 2009; 25: 511-6.

66. Prudhomme O’Meara W, Smith DL, McKenzie FE. Potential Impact of Intermittent Preventive Treatment (IPT) on Spread of Drug-Resistant Malaria. PLoS Med. 2006; 3: e141.

67. Le Menach A, Takala S, McKenzie FE, Perisse A, Harris A, Flahault A, Smith DL. An elaborated feeding cycle model for reductions in vectorial capacity of night-biting mosquitoes by insecticide-treated nets. Malar J. 2007; 6: 10.

68. Killeen GF, Smith TA. Exploring the contributions of bed nets, cattle, insecticides and excitorepellency to malaria control: a deterministic model of mosquito host-seeking behaviour and mortality. Trans R Soc Trop Med Hyg. 2007; 101: 867-80. 